\title{
Article
}

\section{Kinetic and Thermodynamic Approaches for the Efficient Formation of Mechanical Bonds}

William R. Dichtel, Ognjen S\#. Miljanic\#, Wenyu Zhang, Jason M. Spruell, Kaushik Patel, Ivan Aprahamian, James R. Heath, and J. Fraser Stoddart

Acc. Chem. Res., 2008, 41 (12), 1750-1761 • DOI: 10.1021/ar800067h • Publication Date (Web): 07 October 2008

Downloaded from http://pubs.acs.org on January 9, 2009

\section{More About This Article}

Additional resources and features associated with this article are available within the HTML version:

- $\quad$ Supporting Information

- Access to high resolution figures

- $\quad$ Links to articles and content related to this article

- Copyright permission to reproduce figures and/or text from this article

\section{View the Full Text HTML}




\title{
ACCOUNTS \\ of chemical research
}

\section{Kinetic and Thermodynamic Approaches for the Efficient Formation of Mechanical Bonds}

\author{
WILLIAM R. DICHTEL, ${ }^{\dagger, \neq}$ OGNJEN Š. MILJANIĆ, ${ }^{+}$ \\ WENYU ZHANG, ${ }^{+}$JASON M. SPRUELL, $^{+, \S}$ KAUSHIK PATEL, $^{+, \S}$ \\ IVAN APRAHAMIAN, ${ }^{+}$JAMES R. HEATH, ${ }^{*, \neq}$ AND \\ J. FRASER STODDART ${ }^{*,+, \S}$ \\ ${ }^{t}$ Department of Chemistry and Biochemistry, University of California, Los \\ Angeles, 405 Hilgard Avenue, Los Angeles, California 90095, "\#Division of \\ Chemistry and Chemical Engineering, California Institute of Technology, \\ 1200 East California Boulevard, Pasadena, California 91125, ${ }^{\S}$ Department of \\ Chemistry, Northwestern University, 2145 Sheridan Road,
}

Evanston, Illinois 60208

RECEIVED ON MARCH 3, 2008

\section{CON SPECTUS}

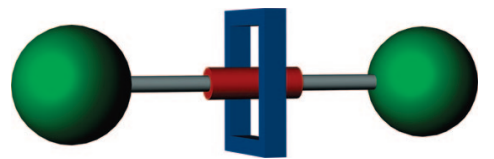

A mong the growing collection of molecular systems under consideration for nanoscale device applications, mechanically interlocked compounds derived from electrochemically switchable bistable [2]rotaxanes and [2]catenanes show great promise. These systems demonstrate dynamic, relative movements between their components, such as shuttling and circumrotation, enabling them to serve as stimuli-responsive switches operated via reversible, electrochemical oxidation-reduction rather than through the addition of chemical reagents. Investigations into these systems have been intense for a number of years, yet limitations associated with their synthesis have hindered incorporation of their mechanical bonds into more complex architectures and functional materials.

We have recently addressed this challenge by developing new template-directed synthetic protocols, operating under both kinetic and thermodynamic control, for the preparation of bistable rotaxanes and catenanes. These methodologies are compatible with the molecular recognition between the $\pi$-electron-accepting cyclobis(paraquat-p-phenylene) (CBPQT ${ }^{4+}$ ) host and complementary $\pi$-electron-donating guests. The procedures that operate under kinetic control rely on mild chemical transformations to attach bulky stoppering groups or perform macrocyclizations without disrupting the host-guest binding of the rotaxane or catenane precursors. Alternatively, the protocols that operate under thermodynamic control utilize a reversible ring-opening reaction of the $\mathrm{CBPQT}^{4+}$ ring, providing a pathway for two cyclic starting materials to thread one another to form more thermodynamically stable catenaned products. These complementary pathways generate bistable rotaxanes and catenanes in high yields, simplify mechanical bond formation in these systems, and eliminate the requirement that the mechanical bonds be introduced into the molecular structure in the final step of the synthesis.

These new methods have already been put into practice to prepare previously unavailable rotaxane architectures and novel complex materials. Furthermore, the potential for utilizing mechanically interlocked architectures as device components capable of information storage, the delivery of therapeutic agents, or other desirable functions has increased significantly as a result of the development of these improved synthetic protocols.

\section{Introduction}

The mechanical bonds and noncovalent forces that hold together the separate components in mechanically interlocked molecules give rise to relative motions, such as circumrotation and shuttling, which can be used in solid-state devices, for example, ultradense molecular memory circuits. ${ }^{1}$ Bistable [2] rotaxanes and [2]catenanes, which incorporate the cyclobis(paraquat-p-phenylene) cyclophane $\mathrm{CBPQT}^{4+}$ as a $\pi$-electron-accepting ring component, are particularly well suited to these applications, because 
their switching actions can be controlled by the reversible oxidation of their $\pi$-electron-donating primary recognition sites, for example, tetrathiafulvalene (TTF). This feature allows these bistable molecules to be switched across a range of different environments, without the addition of chemical reagents. Since their emergence ${ }^{2}$ almost two decades ago, virtually every member of these classes of compounds has been synthesized using kinetically controlled "clipping" approaches (Figure 1) in which a partially formed $\mathrm{CBPQT}^{4+}$ ring recognizes a dumbbell or macrocycle containing complementary $\pi$-electron-rich recognition unit. This recognition process templates the final bond-forming step, which results in closure of the ring. Moderate yields, long reaction times, operationally challenging reaction conditions (12 kbar pressure), and the incompatibility of the $\mathrm{CBPQT}^{4+}$ ring to most subsequent chemical transformations limit the practical utility of this approach to the preparation of bistable [2]rotaxanes and [2]catenanes. These shortcomings represent serious challenges to the further development of increasingly sophisticated donor-acceptor mechanically interlocked compounds.
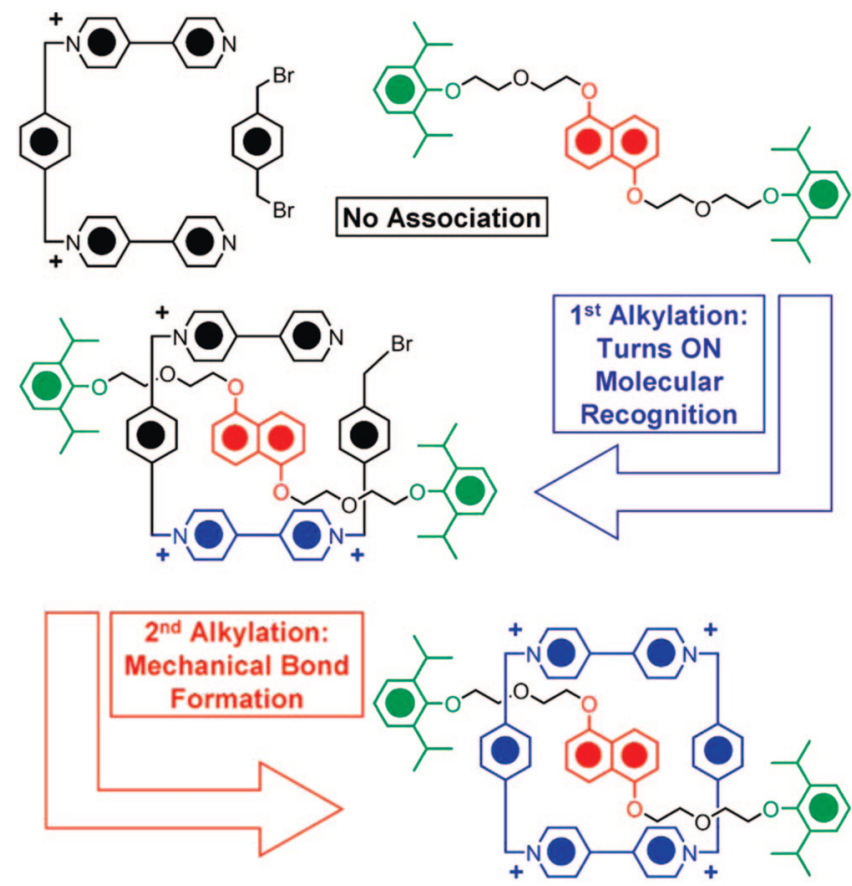

FIGURE 1. The template-directed clipping reaction used historically for the formation of $\mathrm{CBPQT}^{4+}$ rings and mechanical bonds in donor-acceptor rotaxanes (and catenanes).

A key realization in the establishment of improved synthetic protocols for these compounds (Figure 2) is that the noncovalent bonding interactions, which template their synthesis, live on in the final structures and, indeed, are crucial for their subsequent function. Thus, kinetically controlled reactions, where these stabilizing forces are retained in the transition

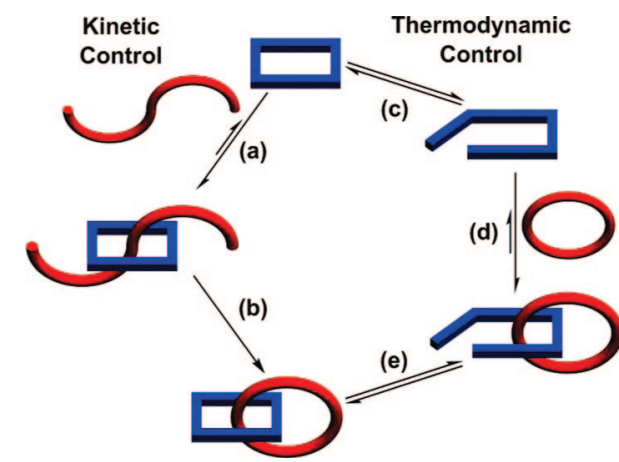

FIGURE 2. Both kinetic (left) and thermodynamic (right) control can be exercised in the catenanes synthesis. Kinetically controlled reactions proceed through (a) pseudorotaxane formation, followed by (b) an irreversible ring closure. Thermodynamically controlled methods rely on (c) reversible ring opening of the $\mathrm{CBPQT}^{4+}$ cyclophane, followed by the (d) coordination to a crown ether, and finally (e) reversible ring closure.

state, can be expected to give mechanically interlocked compounds in high yields. Furthermore, these compounds are lower in free energy than their nonmechanically interlocked components, suggesting that their synthesis under thermodynamic control should also be highly efficient. This approach employs reversible reactions to ultimately provide the most thermodynamically stable products, even if less stable side products are formed during the course of the reaction. Donor-acceptor mechanically interlocked compounds had not been synthesized previously under these conditions, which offer unique advantages relative to kinetically controlled approaches, such as the dynamic exchange of less stable mechanical bonds for more stable ones.

Our recent success in developing both types of synthetic methodologies is a direct result of our investigations of mild chemical transformations compatible with the $\mathrm{CBPQT}^{4+}$ ring and its host-guest complexes. This Account describes the development of new kinetically and thermodynamically controlled protocols for the preparation of donor-acceptor rotaxanes and catenanes and the use of these new protocols for the incorporation of mechanical bonds into increasingly complex molecules.

\section{Highly Efficient Kinetically Controlled Syntheses of [2]Rotaxanes}

The CBPQT ${ }^{4+}$ cyclophane binds $\pi$-electron-rich aromatic systems, such as 1,5-dioxynaphthalene (DNP) and tetrathiafulvalene (TTF) with high association constants $\left(K_{\mathrm{a}} \approx 10^{5} \mathrm{M}^{-1}\right.$ for oligo(ethylene glycol)-bearing derivatives). ${ }^{3}$ Consequently, the 1:1 complexes are the dominant species in DMF or MeCN solutions containing the cyclophane and a DNP or TTF derivative at concentrations (1-200 mM) used typically in prepar- 
SCHEME 1. Template-Directed Synthesis of the [2]Rotaxane $3 \cdot 4 \mathrm{PF}_{6}$ Using the CuAAC Methodology

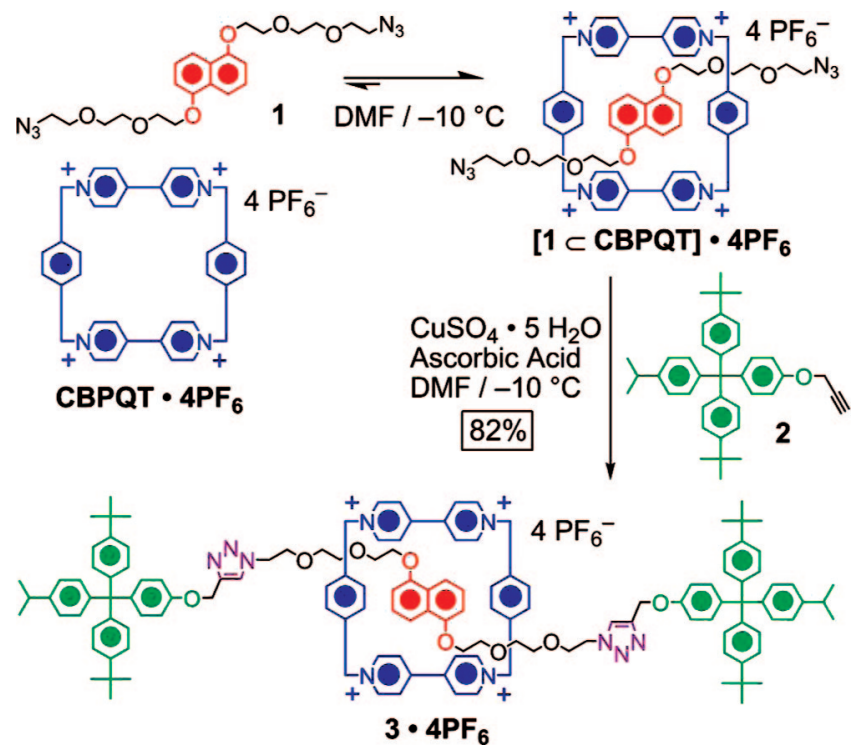

ative procedures. Hence, synthetic transformations that are sufficiently mild so as to be compatible with the complex formation can, in principle, be used either (i) to attach bulky stoppers, affording rotaxanes, or (ii) to effect macrocylizations, affording catenanes, both in high yield. Until recently, few such transformations were known, largely because of the sensitivity of the CBPQT ${ }^{4+}$ ring toward nucleophiles and bases. We have found, however, that mild conditions, excellent functional group tolerance, complete regioselectivity, and the high efficiency of the $\mathrm{Cu}(\mathrm{I})$-catalyzed azide-alkyne cycloaddition ${ }^{4,5}$ (CUAAC) make it an ideal reaction for the formation of mechanical bonds under kinetic control. ${ }^{6}$ The protocol is exemplified (Scheme 1) by the synthesis of the [2]rotaxane 3. $4 \mathrm{PF}_{6}$. The cyclophane $\mathbf{C B P Q T} \cdot 4 \mathrm{PF}_{6}$ is threaded by the DNP derivative $\mathbf{1}$, bearing two triethyleneglycol chains terminated by azide groups, forming the pseudorotaxane $[\mathbf{1} \subset \mathbf{C B P Q T}] \cdot 4 \mathrm{PF}_{6}$. The addition of the bulky propargyl ether $\mathbf{2}$ with its tetraarylmethane core, along with catalytic amounts of $\mathrm{CuSO}_{4} \cdot 5 \mathrm{H}_{2} \mathrm{O}$ and ascorbic acid, resulted in the efficient formation of the [2]rotaxane $\mathbf{3} \cdot 4 \mathrm{PF}_{6}$. Notably, the formation of the corresponding dumbbell, the CUAAC product without the encircling $\mathrm{CBPQT}^{4+}$ ring, was not observed, even when only a slight excess of $\mathbf{C B P Q T}^{4+}$ (1.05 equiv relative to $\mathbf{1}$ ) was employed in the reaction. No doubt inspired by the increasing use of the CUAAC reaction in materials science ${ }^{7}$ and biochemistry, ${ }^{8}$ several researchers have used this approach successfully to prepare [2]rotaxanes of other kinds. ${ }^{9}$ A particularly elegant example is the one described by Leigh and co-workers ${ }^{10}$ in which the $\mathrm{Cu}(\mathrm{l})$ ions serve both as catalytic and templating elements in the formation of the rotaxane.

\section{Syntheses of [n]Catenanes under Kinetic and Thermodynamic Control}

A similar kind of "click chemistry" strategy (Figure 2a,b) was employed in the synthesis (Scheme 2) of [2]catenanes, ${ }^{11}$ simply by performing a macrocyclization of a DNP derivative, whose oligo(ethylene glycol) chains are terminated at one end by an alkyne and at the other by an azide in the presence of CBPQT $^{4+}$. The efficiency of the macrocyclization was investigated $^{12}$ as a function of ring size by subjecting the [2]pseudorotaxanes [4a-c $\subset \mathbf{C B P Q T}] \cdot 4 \mathrm{PF}_{6}$ to either the conditions employed for the synthesis of $\mathbf{3} \cdot 4 \mathrm{PF}_{6}$ or $\mathrm{Cul}$ in $\mathrm{MeCN}$. These conditions gave the corresponding [2]catenanes $\mathbf{5 b} \cdot \mathbf{4} \mathrm{PF}_{6}$ and 5c. $4 \mathrm{PF}_{6}$ in $41 \%$ and $23 \%$ isolated yields, respectively. The [2]pseudorotaxane [4a $\subset \mathbf{C B P Q T}] \cdot 4 \mathrm{PF}_{6}$, however, failed to react, and only $\mathbf{4 a}$ and $\mathbf{C B P Q T} \cdot 4 \mathrm{PF}_{6}$ were recovered, possibly because the glycol chains of $\mathbf{4 a}$ are too short to form a macrocycle free of significant ring strain.

SCHEME 2. Template-Directed Synthesis of Donor-Acceptor [2]Catenanes $\mathbf{5 a}-\mathbf{c} \cdot 4 \mathrm{PF}_{6}$ and $\mathbf{7 a}-\mathbf{c} \cdot 4 \mathrm{PF}_{6}$ with Varying Sizes of the DNP-Containing Macrocycles Using Either the CuAAC or Eglinton Oxidative Alkyne Homocoupling
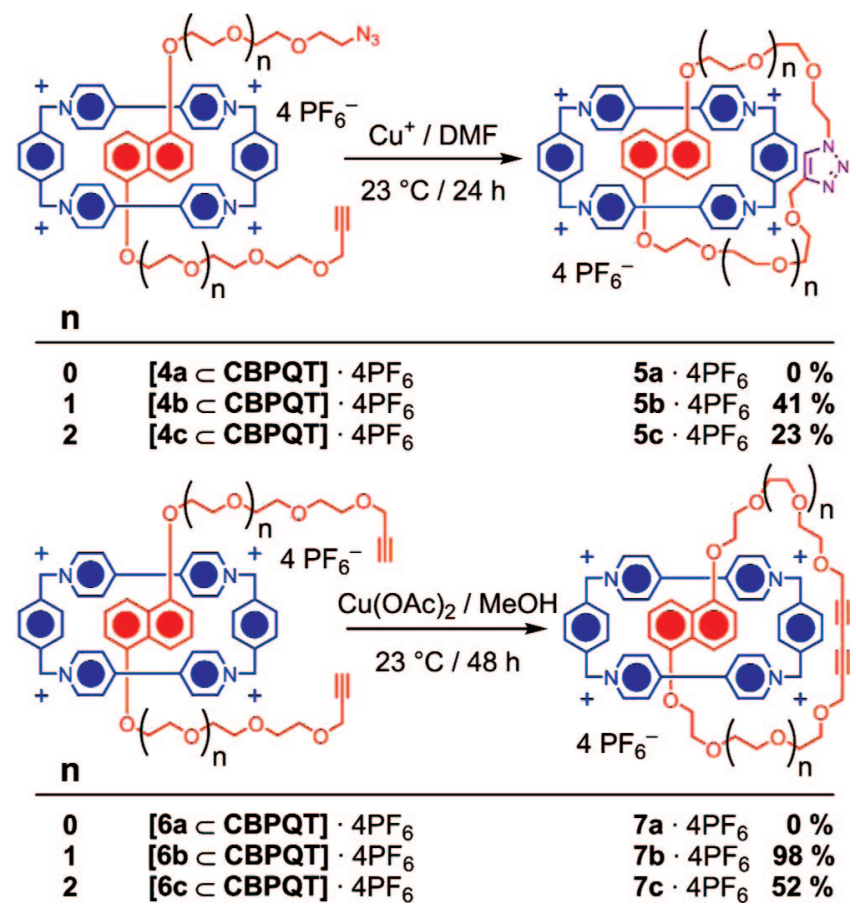

The successful syntheses of the [2]rotaxane $3 \cdot 4 \mathrm{PF}_{6}$ and the catenanes $\mathbf{5 b} \cdot \mathbf{4} \mathrm{PF}_{6}$ and $\mathbf{5} \mathbf{c} \cdot 4 \mathrm{PF}_{6}$ demonstrate that the copper acetylides, which most likely serve as intermediates in the CUAAC reaction, are compatible with the chemically sensitive CBPQT $^{4+}$ and its pseudorotaxane-like host-guest complexes. We have also investigated the likelihood of [2]catenane formation employing other reactions, such as the Eglinton oxidative alkyne homocoupling, ${ }^{13}$ which also proceed through 
Cu-acetylide intermediates. In addition to the more symmetrical nature of the starting materials and products, we found the same trends in yields relative to the oligo(ethylene glycol) chain lengths during the formation of the catenanes $\mathbf{7 a}-\mathbf{c} \cdot 4 \mathrm{PF}_{6}$, as was observed for those using the CuAAC approach. The dialkyne-containing catenanes, however, were formed in significantly improved yields, as exemplified most notably in the synthesis of $\mathbf{7} \mathbf{b} \cdot 4 \mathrm{PF}_{6}$, which was isolated in 97\% yield, reflecting a remarkably efficient macrocyclization process. As observed in the case of $[\mathbf{4 a} \subset \mathbf{C B P Q T}] \cdot 4 \mathrm{PF}_{6}$, no catenane was obtained during the attempted cyclization of the pseudorotaxane $[\mathbf{6 a} \subset \mathbf{C B P Q T}] \cdot 4 \mathrm{PF}_{6}$ : a single-crystal X-ray structural analysis of this pseudorotaxane also suggested that the oligo(ethylene glycol) chains of $\mathbf{6 a}$ are far too short to form a strain-free macrocycle.

The noncovalent bonding interactions between the components of catenanes and rotaxanes stabilize these molecules significantly relative to the sum of their individual components lacking mechanical bonds. This pronounced energetic bias renders dynamic covalent chemistry (DCC $)^{14,15}$ appealing for the synthesis (Figure $2 \mathrm{c}-\mathrm{e}$ ) of mechanically interlocked compounds. DCC depends on thermodynamically controlled reactions that recycle the starting materials and the side products until equilibria favoring the mechanically interlocked compounds as the most stable ones are established. We have shown ${ }^{16}$ recently that a thermodynamically controlled nucleophilic substitution can provide an efficient route to donor-acceptor catenanes incorporating the $\mathrm{CBPQT}^{4+}$ ring. In this research, tetrabutylammonium iodide (TBAI) was employed as the catalyst in a "magic ring" experiment, in which the $\mathrm{CBPQT}^{4+}$ ring was first of all opened and then reversibly closed around a crown ether ring to form a donor-acceptor catenane. Mechanistically, this transformation commences (Scheme 3) with a rate-limiting nucleophilic attack of TBAI onto the $\mathrm{CBPQT}^{4+}$ ring to generate the trication $\mathbf{8}^{3+}$. TBAI was chosen as the catalyst because it is a good nucleophile, a good leaving group, a poor reducing agent, and soluble in MeCN. The tricationic $\pi$-acceptor $\mathbf{8}^{3+}$ complexes readily ${ }^{17}$ with crown ethers such as $\mathbf{9 a}-\mathbf{c}$ to give pseudorotaxanes $[\mathbf{8} \subset \mathbf{9}]^{3+}$. Nucleophilic attack then occurs, closing the $\mathrm{CBPQT}^{4+}$ ring around the crown ethers to give catenanes $10 \mathbf{a}-\mathbf{c}^{4+}$, regenerating the iodide ion. The reversibility of the overall reaction was confirmed by an exchange experiment. The preformed [2]catenane $\mathbf{1 0} \mathbf{a}^{4+}$ was treated with an excess of the crown ether $\mathbf{9 b}$, which has been shown to associate more strongly with $\mathbf{C B P Q T}^{4+}$ and presumably with the trication $\mathbf{8}^{3+}$. After equilibrium was reached over the course of 11
SCHEME 3. Mechanism for the Template-Directed Synthesis of Various Donor-Acceptor [2]Catenanes $\left(\mathbf{1 0 a}-\mathbf{c}^{4+}\right)$ under Thermodynamic Control

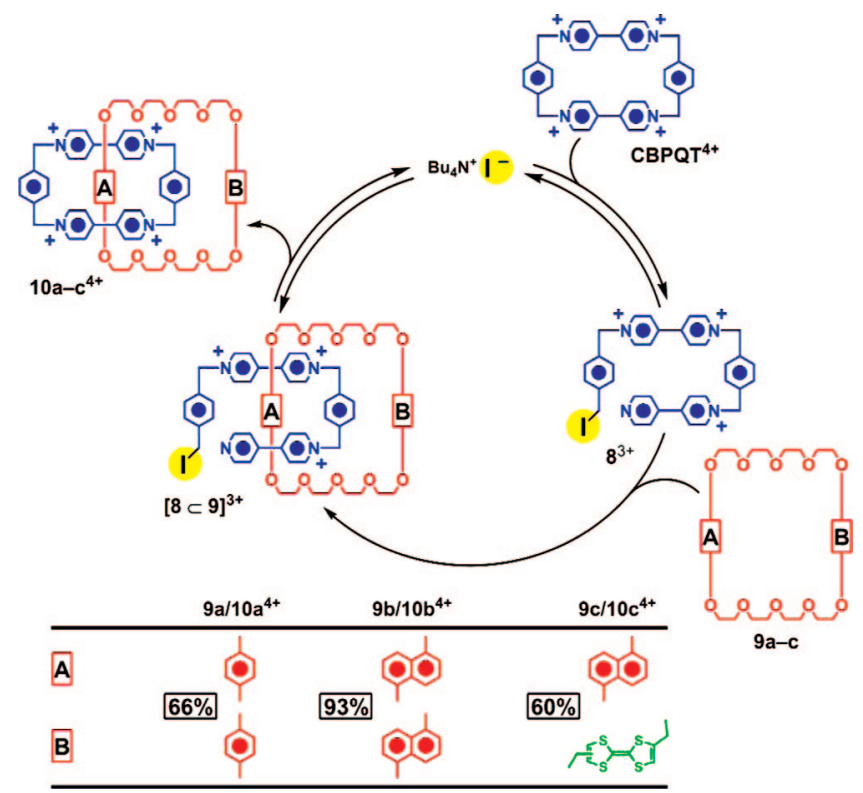

days, the more stable [2]catenane $\mathbf{1 0} \mathbf{b}^{4+}$ constituted about $\sim 90 \%$ of the final reaction mixture.

Since the reaction proceeds only at elevated temperatures $\left(\sim 80^{\circ} \mathrm{C}\right)$, the equilibration can be arrested on cooling to room temperature, a property that allows the convenient isolation of the catenated products. The approach is general in scope, is easy to execute (since protecting atmospheres and dry solvents are not required), and proceeds in yields as high as $93 \%$. In the context of molecular devices, the bistable catenane $\mathbf{1 0} \mathbf{c}^{4+}$ was prepared in a straightforward manner by dynamic nucleophilic substitution in $60 \%$ yield, that is, almost three times more efficiently than by using ${ }^{18}$ the traditional kinetically controlled approach. We have, however, failed thus far to extend this method to the synthesis of rotaxanes. Our rationalization for this failure is that at the elevated temperatures required for reaction to occur, the association of the $\pi$-electron-accepting $\mathbf{8}^{3+}$ with an acyclic $\pi$-donating dumbbell is less favorable compared with the association of $\mathbf{8}^{3+}$

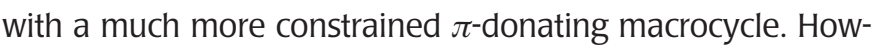
ever, on going from the CBPQT ${ }^{4+}$ ring to cyclobis(paraquat4,4'-biphenylene), a larger $\pi$-accepting ring that associates ${ }^{19}$ with crown ethers with 1:2 stoichiometry, we were able to prepare $^{20}$ the respective [3]catenanes, derived from $\mathbf{9 a}$ and $\mathbf{9 b}$, in yields greater than $84 \%$ and $91 \%$, respectively. This virtually undiminished efficiency of conversion suggests that even larger mechanically interlocked systems, including perhaps the elusive donor-acceptor polycatenanes, could be targeted by the protocol involvolving dynamic nucleophilic substitution. 


\section{Formation of Higher Order Rotaxanes Using CuAAC}

One of the most significant challenges toward realizing the long-standing objective to incorporate multiple $\mathrm{CBPQT}^{4+}$ rings into higher order rotaxane architectures and ultimately into a wide variety of materials is the modest efficiency of the clipping reaction and the low convergence that characterized the strategy associated with its synthesis. For example, the simultaneous clipping of two $\mathrm{CBPQT}^{4+}$ rings onto a bistable dumbbell afforded ${ }^{21}$ only $9 \%$ of the desired [3]rotaxane, which represents one of the most complex compounds of this kind that can be prepared practically using this synthetic approach. No longer constrained by the requirement of performing the clipping reaction on a fully formed dumbbell template in the final step of the synthesis, retrosynthetic analysis of a palindromic [3]rotaxane structure suggested a disconnection in the middle of the molecule, with the simultaneous formation of the final structure and incorporation of the $\mathrm{CBPQT}^{4+}$ rings using the CuAAC reaction. Indeed, a similar, albeit simplified, palindromic [3]rotaxane, 13.8PF ${ }_{6}$, was synthesized ${ }^{6}$ (Scheme 4) from the stoppered DNP azide 11, the bis(propargyl ether) 12, and CBPQT - $4 \mathrm{PF}_{6}$ in a significantly improved $79 \%$ isolated yield. Furthermore, by substitution of tris-1,3,5(4'-ethynylphenyl)benzene $\mathbf{1 4}$ for $\mathbf{1 2}$, the branched [4]rotaxane $15 \cdot 12 \mathrm{PF}_{6}$ was obtained in $72 \%$ isolated yield and represents the first example of a donor-acceptor [4]rotaxane prepared in our laboratory. The good yields obtained during the synthesis of these higher order rotaxanes point to a significant advantage of the CUAAC stoppering approach, since there is essentially no penalty in the yields of the compounds when incorporating multiple cyclophanes into the molecules.

We have subsequently elaborated this methodology to enable the one-pot synthesis of rotaxanes with two different stoppers, employing sequential CUAAC reactions. ${ }^{22}$ The order of the two cycloadditions is controlled by protecting the alkyne functions with trimethylsilyl (TMS) groups, which are then removed after the first CuAAC reaction is complete. Though methods commonly employed for alkyne desilylation occur under more strongly nucleophilic conditions and are thus not compatible with the presence of the $\mathrm{CBPQT}^{4+}$ ring, we confirmed that $\mathrm{Ag}(\mathrm{l})$-catalyzed hydrolysis of the TMS groups ${ }^{23}$ is compatible with both the presence of the $\mathrm{CBPQT}^{4+}$ ring and carrying out the subsequent CuAAC reactions in the same pot. In order to highlight the efficiency of this methodology, the 3 -fold symmetric amphiphilic [4]rotaxane $\mathbf{2 1} \cdot 12 \mathrm{PF}_{6}$ was synthesized ${ }^{24}$ (Scheme 5) in one-pot, by (a) performing the CuAAC reaction between the monofunctional DNP derivative $\mathbf{1 7}$ and the triazide 16, (b) the removal of the silyl protecting groups, and (c) the second stoppering reaction with the hydrophilic azide $\mathbf{2 0}$ in the presence of the $\mathrm{CBPQT}^{4+}$ ring. The complexity of the final products available from comparatively simple starting mate-

SCHEME 4. Template-Directed Synthesis of the [3]- and [4]Rotaxanes $\mathbf{1 3} \cdot 8 \mathrm{PF}_{6}$ and $\mathbf{1 5} \cdot 12 \mathrm{PF}_{6}$ through CuAAC of a Stoppered DNP-Derivative with Bi- and Trifunctional Alkynes

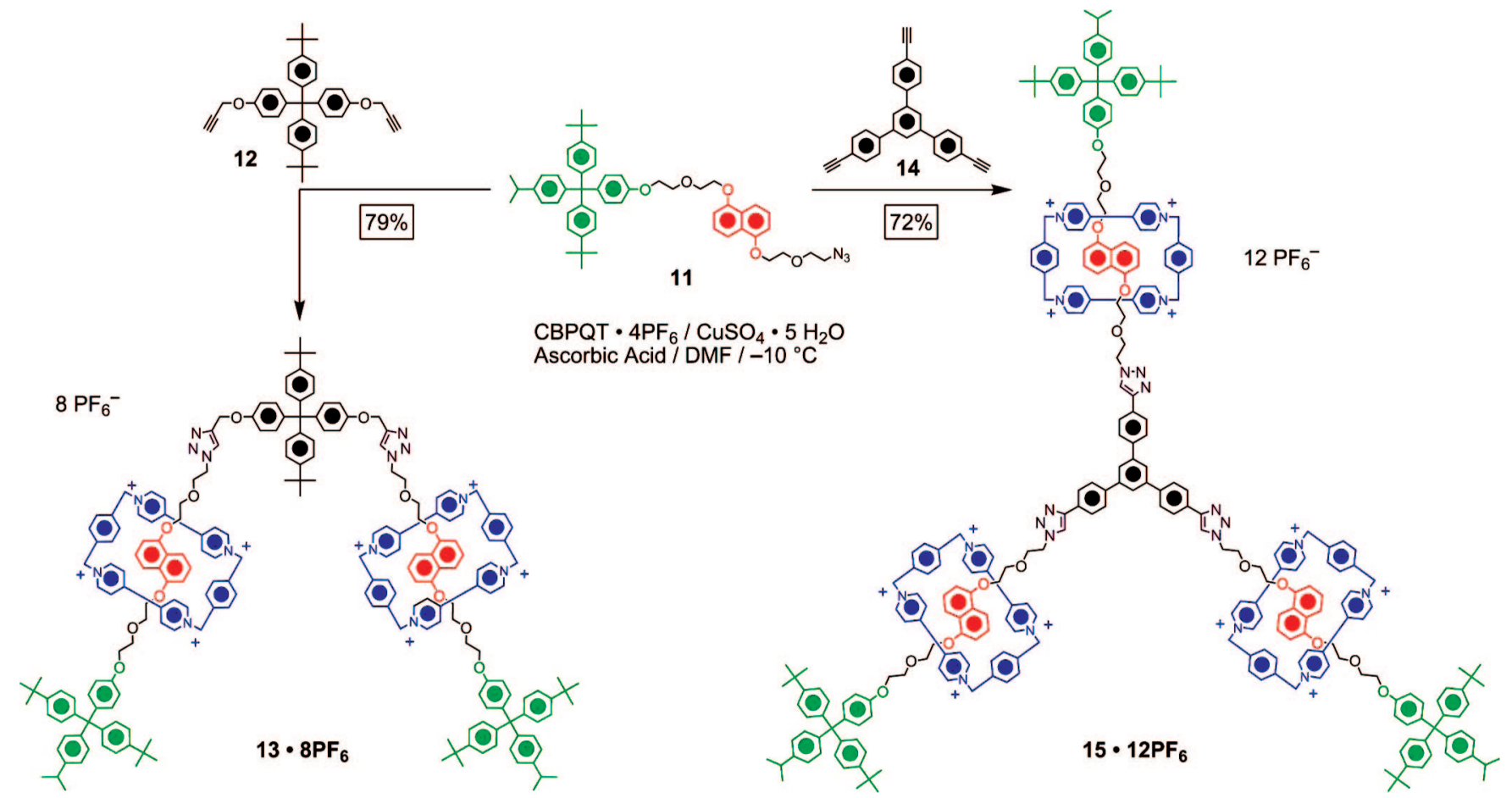


SCHEME 5. One-Pot Sequential Template-Directed Synthesis of an Amphiphilic [4]Rotaxane 21 $12 \mathrm{PF}_{6}$

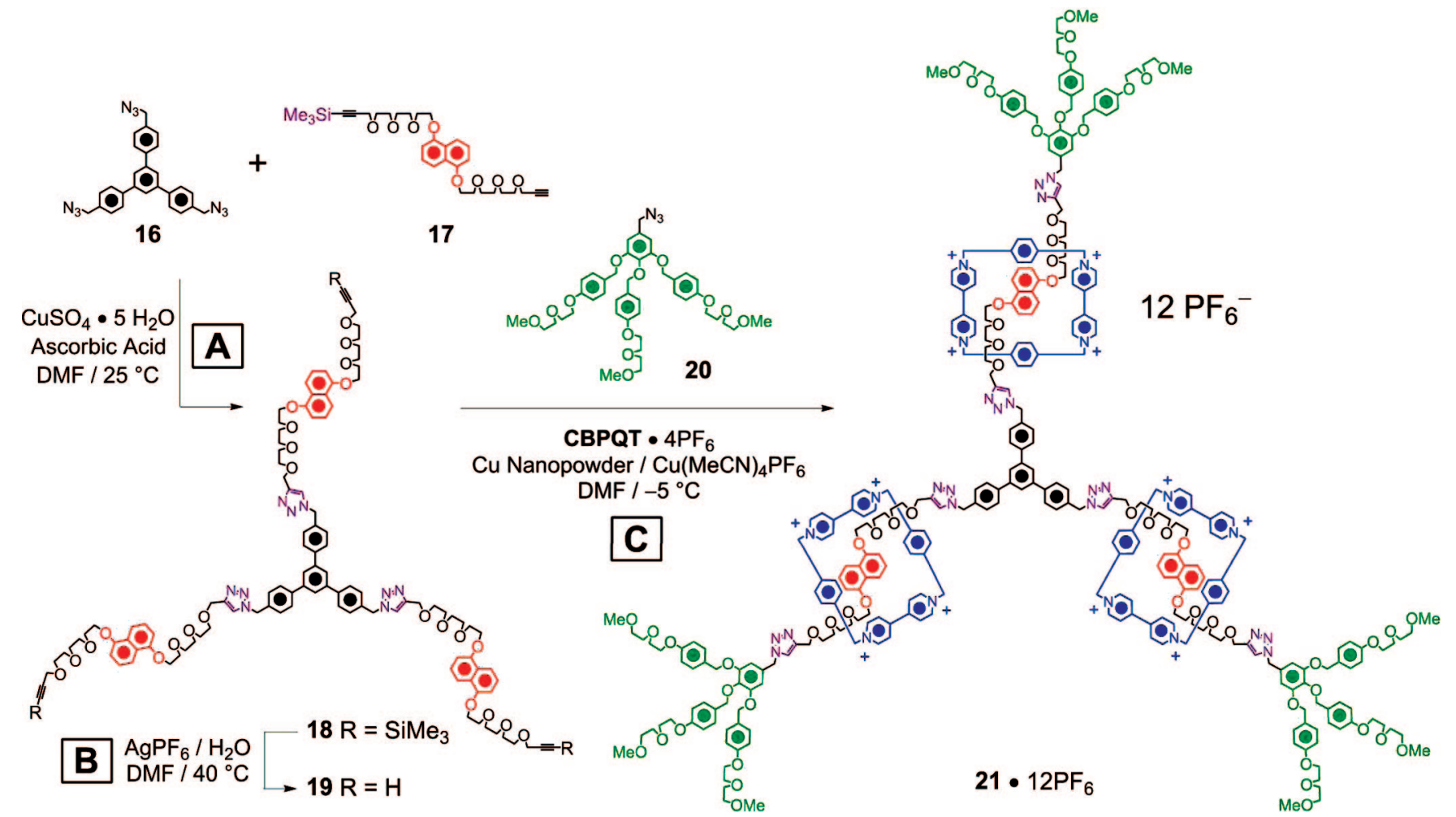

rials using this strategy is noteworthy, and as a consequence of identifying mild and efficient desilylation conditions, the introduction of the $\mathrm{CBPQT}^{4+}$ ring need not occur in the final step of the synthesis of the rotaxane. We are currently taking advantage of these features to design and synthesize previously unavailable topologies of mechanically interlocked donor-acceptor compounds.

\section{Shuttling and Switching within Triazole- Containing [2]Rotaxanes}

An important aspect of the development of the CuAAC threading-followed-by-stoppering synthetic approach involved an investigation of whether the incorporation of disubstituted 1,2,3-triazole units into the dumbbell components of [2]rotaxanes would impact significantly either (i) the thermally activated or (ii) electrochemically controlled motions of the $\mathrm{CBPQT}^{4+}$ ring. The former situation was investigated ${ }^{25}$ by measuring (Figure 3) the rate of shuttling of the cyclophane between two degenerate DNP recognition sites separated by a bis(triazole)-containing central spacer unit in the molecular shuttle $\mathbf{2 1} \cdot 4 \mathrm{PF}_{6}$. When the motion of the $\mathrm{CBPQT}^{4+}$ ring is slow on the NMR time scale, the resonances of protons on opposite sides of the dumbbell separate into two signals of equal intensity. See, for example, the resonances for the $t$-Bu $\left(\mathrm{H}_{\mathrm{a}}, \mathrm{H}_{\mathrm{a}}{ }^{\prime}\right)$ and $i-\operatorname{Pr}\left(\mathrm{H}_{\mathrm{b}}, \mathrm{H}_{\mathrm{b}}{ }^{\prime}\right)$ protons in the partial spectrum recorded at $261 \mathrm{~K}$ in Figure 3. The frequency of the shuttling increases with temperature, resulting eventually in the coalescence of the two pairs of signals at $309 \mathrm{~K}$, corresponding to an energy barrier of $15.5 \pm 0.1 \mathrm{kcal} \mathrm{mol}^{-1}$. This value is similar to those measured previously ${ }^{26}$ for degenerate molecular shuttles containing triphenylene and tetra(ethylene glycol) spacers $\left(15.0 \pm 0.2\right.$ and $15.5 \pm 0.1 \mathrm{kcal} \mathrm{mol}^{-1}$, respectively). These findings suggest that the disubstituted 1,2,3-triazole rings may be incorporated into rotaxanes and may even be located between the recognition sites without significant concern that they will interfere with the movement of the $\mathrm{CBPQT}^{4+}$ ring.

SCHEME 6. Template-Directed CuAAC-Mediated Synthesis of an Electrochemically Switchable Bistable [2]Rotaxane $\mathbf{2 3} \cdot 4 \mathrm{PF}_{6}$

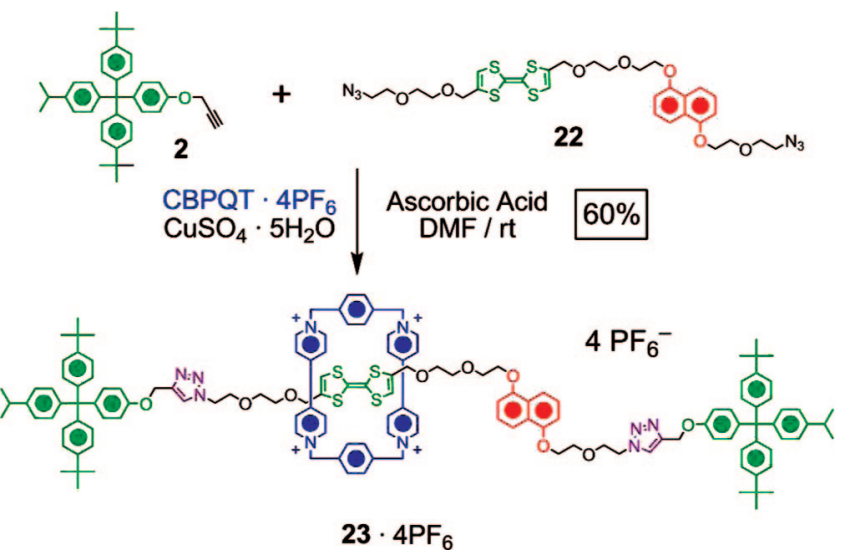



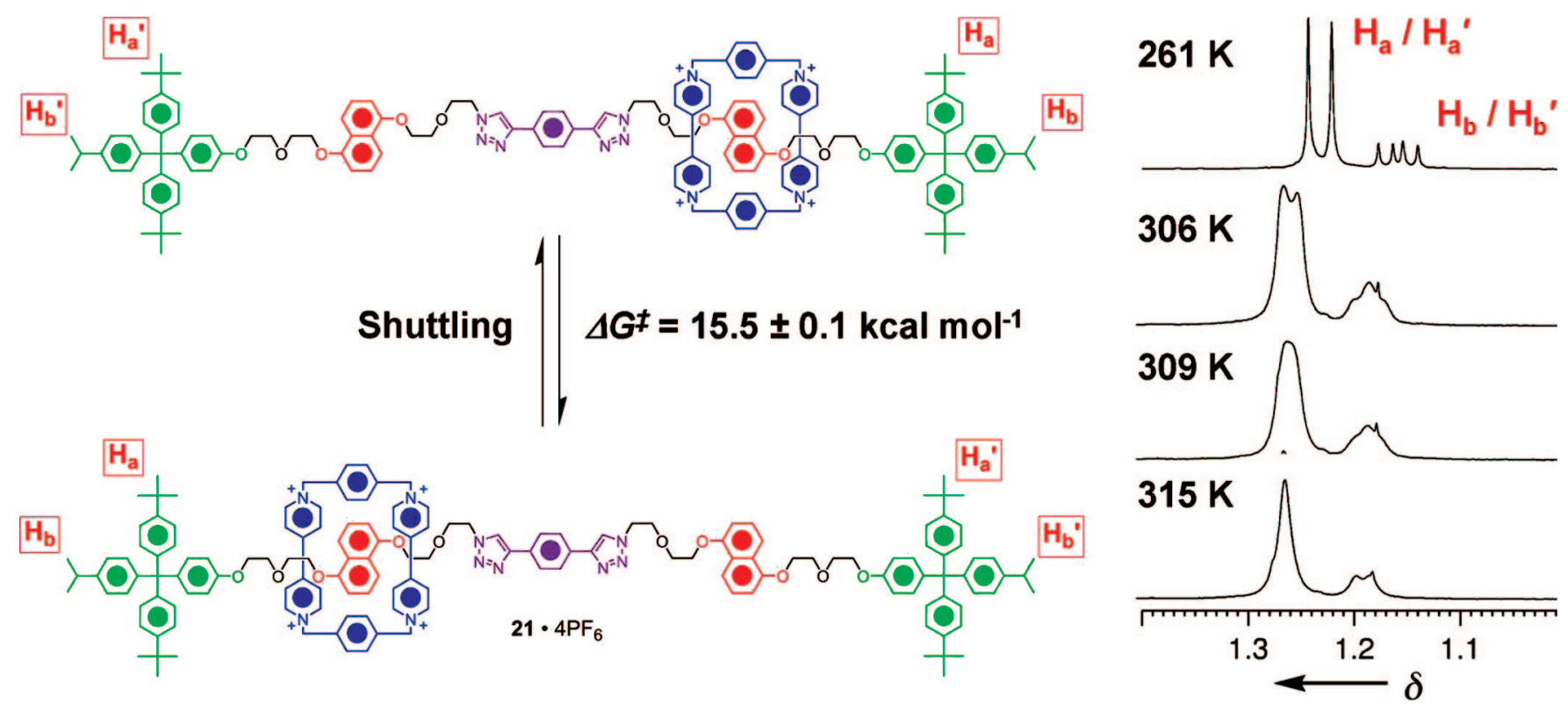

FIGURE 3. The two degenerate co-conformations of the $\mathbf{2 1} \cdot 4 \mathrm{PF}_{6}$ shuttle (left) and portions of the variable-temperature ${ }^{1} \mathrm{H}$ NMR spectra $\left(500 \mathrm{MHz}, \mathrm{CD}_{3} \mathrm{COCD}_{3}\right)$ obtained at different temperatures containing the $t-\mathrm{Bu}\left(\mathrm{H}_{\mathrm{a}} / \mathrm{H}_{\mathrm{a}}{ }^{\prime}\right)$ and $i-\mathrm{Pr}\left(\mathrm{H}_{\mathrm{b}} / \mathrm{H}_{\mathrm{b}}{ }^{\prime}\right)$ resonances.

Satisfied that the disubstituted 1,2,3-triazole units do not disrupt the thermally activated shuttling of the $\mathrm{CBPQT}^{4+}$ ring, we went on to assess their compatibility with the electrochemical switching processes of bistable [2]rotaxanes. The prototypical bistable [2]rotaxane $\mathbf{2 3} \cdot 4 \mathrm{PF}_{6}$ was prepared (Scheme 6) through CuAAC-mediated stoppering of the diazide $\mathbf{2 2}$ containing both a TTF and a DNP moiety. ${ }^{27}$ The ${ }^{1} \mathrm{H}$ NMR spectrum of $23 \cdot 4 \mathrm{PF}_{6}$ in $\mathrm{CD}_{3} \mathrm{COCD}_{3}$, in common with those of other bistable [2]rotaxanes of this type, indicated the dominant presence $(>95 \%)$ of the TTF-encircled translational isomer at equilibrium. The reversibility of the electromechanical switching process of $\mathbf{2 3} \cdot 4 \mathrm{PF}_{6}$ is evident (Figure 4) from spectroelectrochemical (SEC) measurements. Before the switching process is initiated ( $E=0$ vs $\mathrm{Ag}$ wire), the absorbance centered at $840 \mathrm{~nm}$ corresponds to the charge transfer (CT) interaction typically observed when a TTF unit is encircled by a

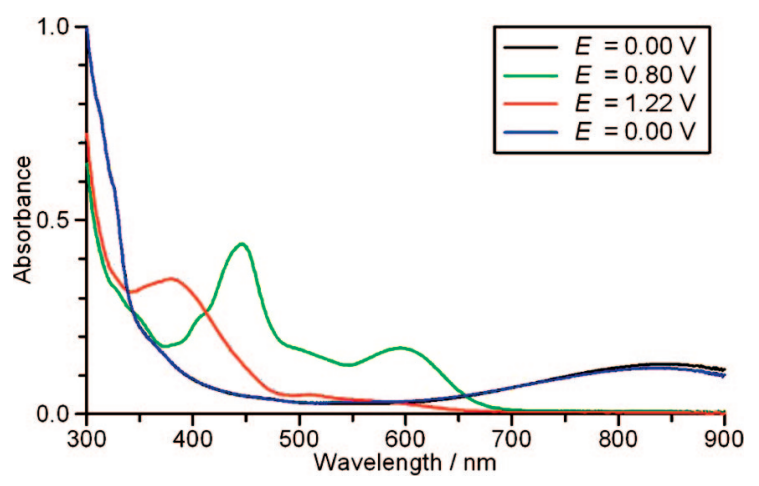

FIGURE 4. UV-visible spectra obtained during the SEC measurements (MeCN, $0.25 \mathrm{mV} \mathrm{s}^{-1}$ scan rate) of the CuAAC-derived bistable [2]rotaxane $\mathbf{2 3} \cdot 4 \mathrm{PF}_{6}$. The applied potential was changed from $E=0 \mathrm{~V}$ to $E=1.22 \mathrm{~V}$ and then back to $E=0 \mathrm{~V}$.
$\mathrm{CBPQT}^{4+}$ ring. Between +0.50 and $+0.80 \mathrm{~V}$, new bands appear with maxima at 445 and $595 \mathrm{~nm}$, corresponding to the absorbance of the TTF radical cation $\left(\operatorname{TTF}^{\circ+}\right)$. These changes are accompanied by the bleaching of the TTF-CBPQT ${ }^{4+} \mathrm{CT}$ band. When the applied potential was increased further, the absorption band of the TTF ${ }^{\circ}$ was replaced by a new peak with a $\lambda_{\max }$ at $380 \mathrm{~nm}$, which can be assigned to the TTF dication $\left(\mathrm{TTF}^{2+}\right)$. In contrast to the TTF* ${ }^{*+}$, the $\mathrm{TTF}^{2+}$ does not absorb significantly between 500 and 600 $\mathrm{nm}$ and so permits the observation of the weak DNP-CBPQT ${ }^{4+}$ CT peak at ca. $530 \mathrm{~nm}$. Finally, when the applied potential is returned to zero, the spectrum gradually makes its way back to its original state containing the TTF-CBPQT ${ }^{4+}$ CT band, verifying the full reversibility of the redox process. These observations, along with other supporting ${ }^{1} \mathrm{H}$ NMR spectroscopic data and the full electrochemical characterization of $\mathbf{2 3} \cdot 4 \mathrm{PF}_{6}$, confirmed that the triazole rings do not interfere with the unique switching mechanisms of these compounds, paving the way for their incorporation into range of new material and device architectures.

\section{Incorporating Mechanical Bonds into Materials and New Environments}

The mechanical force and changes associated with the generation of translational isomers during the electrochemical switching process of these bistable rotaxanes and catenanes form the raison d'etre for their incorporation into molecular electronic devices, nanoelectromechanical systems, and nanoparticle-based controlled release vehicles. These technologi- 

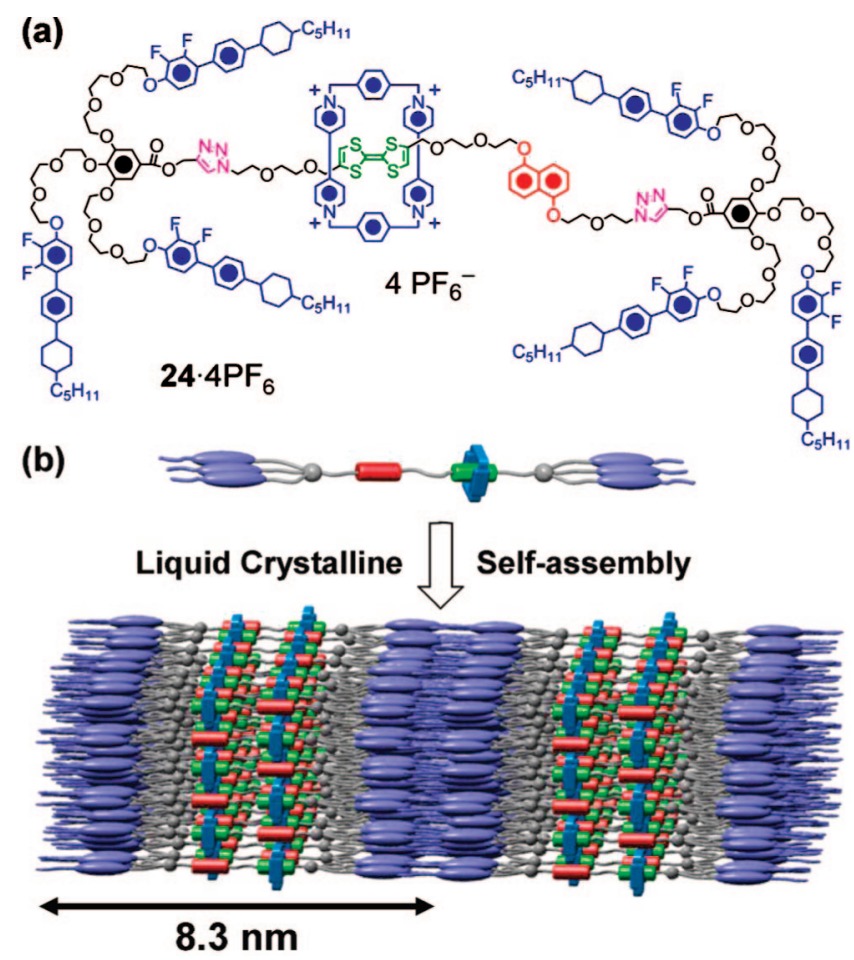

FIGURE 5. (a) Structural formula of a mesogenic electrochemically switchable bistable [2]rotaxane $\mathbf{2 4} \cdot 4 \mathrm{PF}_{6}$ and (b) illustration of the hypothetical organization of $\mathbf{2 4} \cdot 4 \mathrm{PF}_{6}$ into a smectic A phase, as suggested by small-angle $\mathrm{X}$-ray scattering data.

cal applications motivated an investigation of the fundamental thermodynamic and kinetic parameters of the switching processes in a range of condensed environments, such as within polymer gels, ${ }^{28}$ self-assembled monolayers (SAMs) on gold surfaces, ${ }^{29}$ and molecular switch tunnel junctions (MSTJs). ${ }^{3}$ The correlation of these parameters across each of these environments for several bistable rotaxanes and catenanes provides compelling evidence for (i) a universal switching mechanism and (ii) attributing this mechanism to the hysteretic response of molecular memory circuits.

The subsequent development of the CuAAC methodology has greatly enhanced our efforts to prepare new bistable [2]rotaxanes that can operate in different environments, for example, the recent development of a derivative that forms liquid crystalline (LC) phases. ${ }^{30}$ The ordering of liquid crystals is a cooperative phenomenon that is highly sensitive to weak perturbations, including the photoisomerization of small quantities of added molecular rotors. ${ }^{31}$ Hence, we set out to align electrochemically switchable bistable [2]rotaxanes within LC phases as a possible means of amplifying the effect of molecular switching and so influence the bulk LC ordering. The bistable [2] rotaxane $\mathbf{2 4} \cdot \mathbf{4} \mathrm{PF}_{6}$ (Figure 5) with dendritic mesogens as stoppers was synthesized by subjecting the diaz- ide $\mathbf{2 2}$ and mesogens functionalized with propargyl ester groups to the same CUAAC conditions employed for the synthesis of $\mathbf{2 3} \cdot 4 \mathrm{PF}_{6}$. The convergence of the CUAAC approach is particularly noteworthy in this case: it allows $\mathbf{2 2}$ to be employed as a general precursor to nearly all symmetrically stoppered bistable rotaxanes. The high efficiency of the reaction was also critical, because our efforts to attach these mesogens to threads containing TTF and DNP units under esterification conditions had been unsuccessful, precluding even an attempt at synthesizing the mesogenic bistable [2]rotaxane using the clipping protocol. Compound $24 \cdot 4 \mathrm{PF}_{6}$ exhibits a LC smectic $A\left(S_{A}\right)$ phase from $10-150{ }^{\circ} \mathrm{C}$, above which it undergoes thermal decomposition. Peaks with $d$ spacings of 83.0 (100), 41.5 (200), and $27.3 \AA$ (300) were observed in its small-angle X-ray scattering (SAXS) pattern obtained at $40{ }^{\circ} \mathrm{C}$. This layer spacing of $\sim 8 \mathrm{~nm}$ observed for the $S_{A}$ phase corresponds to the extended molecular length of $\mathbf{2 4} \cdot 4 \mathrm{PF}_{6}$. The electrochemical switching of $\mathbf{2 4} \cdot 4 \mathrm{PF}_{6}$, which was characterized in solution by CV and SEC, was qualitatively similar to that shown in Figure 4 for $\mathbf{2 2} \cdot 4 \mathrm{PF}_{6}$. Our current efforts focus on switching LC assemblies of $\mathbf{2 4} \cdot 4 \mathrm{PF}_{6}$ while monitoring its effect on the LC properties of the system as a whole.

In moving beyond using the CUAAC reaction to facilitate the synthesis of well-defined [2]-, [3]-, or [4]rotaxanes, we have recently achieved a long-standing goal of synthesizing donor-acceptor polyrotaxanes that incorporate a considerable number of $\mathrm{CBPQT}^{4+}$ rings. ${ }^{32}$ We are particularly interested in studying the effect of electromechanical switching on the bulk properties of the associated polymer. Relatively few examples of polyrotaxanes have been reported, with the exception of those that incorporate more chemically robust cyclodextrin $^{33}$ or cucurbituril ${ }^{34}$ derivatives as the macrocyclic component. Donor-acceptor polyrotaxanes have remained synthetically elusive, mostly because the clipping reaction is unlikely to provide a high coverage of $\mathrm{CBPQT}^{4+}$ rings onto a stoppered polymer template, that is, dumbbell. We employed a CuAAC step-growth polymerization to prepare $^{32}$ (Scheme 7) azide-terminated polymers $\mathbf{2 7}$ ranging in $M_{\mathrm{n}}$ from 32 to $180 \mathrm{kDa}$ by adjusting the feed ratio of the monomers, bis(triethyleneglycol propargyl ether) DNP derivative $\mathbf{2 5}$ and the DNP diazide $\mathbf{2 6}$. Following purification of the polymers, $\mathrm{CBPQT}^{4+}$ rings were threaded onto the chains, and alkyne-bearing stoppers were finally attached, once again using the CUAAC reaction, providing the polyrotaxane $\mathbf{2 9} \cdot 4 n \mathrm{PF}_{6}$. The use of 0.6 equiv of $\mathbf{C B P Q T}^{4+} \cdot 4 \mathrm{PF}_{6}$ relative to the total number of DNP units resulted in polyrotaxanes with average coverages ranging from $90-58 \%$ of the repeat units 
SCHEME 7. CuAAC-Mediated Polymerization and Subsequent Template-Directed Threading and Stoppering Processes for the Preparation of the Polyrotaxane $\mathbf{2 9} \cdot 4 n \mathrm{PF}_{6}$

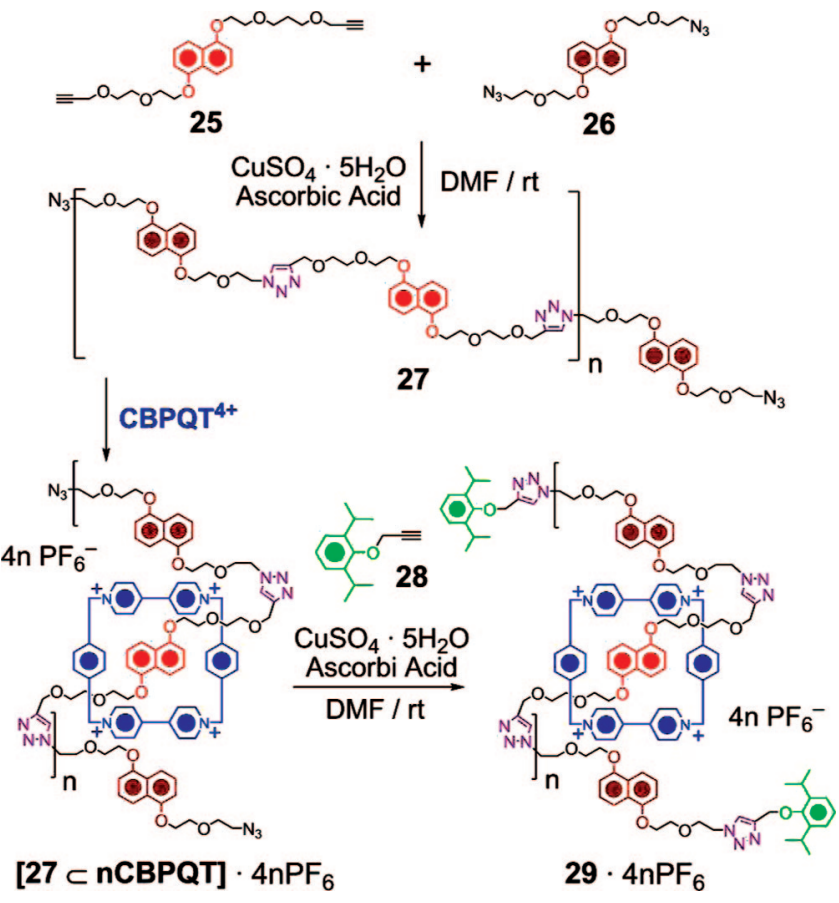

encircled by $\mathrm{CBPQT}^{4+}$ rings, as measured by integration of the appropriate ${ }^{1} \mathrm{H}$ NMR resonances.

One of the first unique properties observed in the polyrotaxanes is that they fold into a compact structure as a consequence of secondary intramolecular interactions. By limiting the $\mathrm{CBPQT}^{4+}$ incorporation to less than $50 \%$ of the available DNP units and using alternating DNP-containing monomers with differing binding affinities, a majority of the rings encircle the more favorable DNP units, while the secondary DNP recognition sites stack on the outside of the rings. In this manner, the polymer adopts a folded conformation (Figure 6), an effect observed previously ${ }^{35}$ in aque-

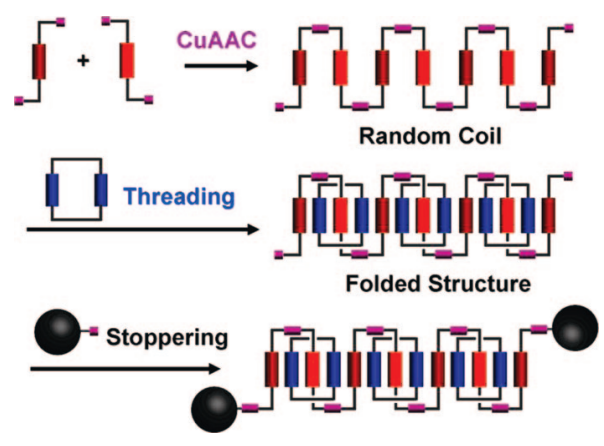

FIGURE 6. Graphical representation of the synthetic approach toward the synthesis of $\mathrm{CBPQT}^{4+}$-containing polyrotaxanes and an idealized representation of the folding observed in these materials as a consequence of stacking of the alongside DNP units against the outside of the $\mathrm{CBPQT}^{4+}$ rings. ous solutions of polymers containing alternating $\pi$-electron donors and acceptors and in more rigid polymers and oligomers of certain polyamide ${ }^{36}$ and $m$-phenylene ethylene $^{37}$ "foldamers". The folding behavior was characterized (Figure 7) by several techniques, such as gel permeation chromatography (GPC), in which the apparent molecular weight of each polyrotaxane sample was smaller than that measured for its polymer thread precursor, a phenomenon that is suggestive of a more compact structure. The noncovalent bonding interactions responsible for the folding process were further characterized by variable-temperature ${ }^{1} \mathrm{H}$ NMR spectroscopy of the polyrotaxanes and an oligomeric model compound. Finally, the lengths of the polyrotaxanes were found to be significantly shorter than the precursor polymeric threads, as measured by atomic force microscopy performed on the polymers drop-cast onto highly ordered pyrolytic graphite. Our current work on these polyrotaxanes focuses on incorporating TTF monomers into the polymer backbone, a modification that will allow study of the electromechanical switching process of the CBPQT ${ }^{4+}$ rings along the polymer backbone, both in solution and in the bulk.

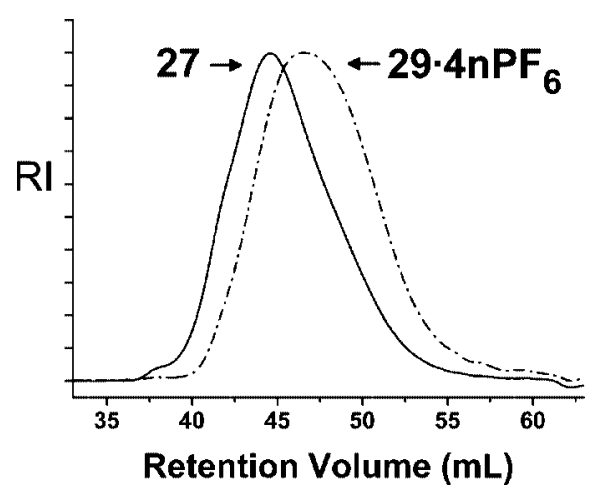

FIGURE 7. Gel permeation chromatographs of the polymer thread $27\left(M_{\mathrm{n}}=32 \mathrm{kDa}\right)$ and its corresponding polyrotaxane $\mathbf{2 9} \cdot 4 n \mathrm{PF}_{6}$, in which an average of $90 \%$ of the repeat units contain a $\mathrm{CBPQT}^{4+}$ ring. Although the molecular weight of $\mathbf{2 9} \cdot 4 n \mathrm{PF}_{6}$ is nearly twice that of $\mathbf{2 7}$, the retention volume of the polyrotaxane increases, a phenomenon that can be attributed to its folded structure.

Finally, inspired by the increasing use of the CuAAC for surface functionalization, ${ }^{38-40}$ we have successfully attached stoppering groups to surface-bound pseudorotaxanes, a simple and direct route to simultaneously synthesizing [2]rotaxanes and interfacing them with nanostructured materials. We have used this approach for the preparation (Figure 8) of snap-top covered silica nano- 


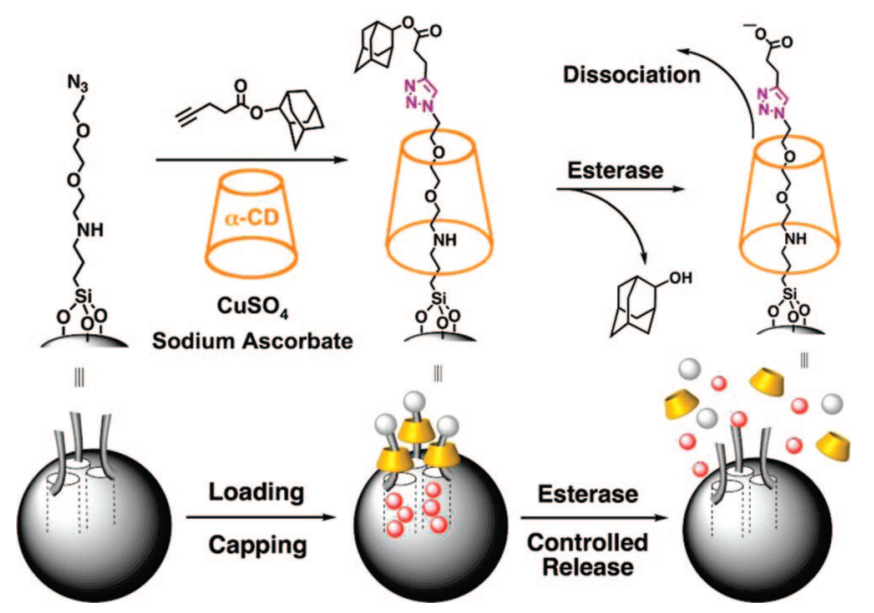

FIGURE 8. The preparation and opening of adamantyl ester stoppered snap-top covered silica nanocontainers. The empty pores of the silica nanoparticles are loaded with the desired payload, such as fluorescent marker rhodamine B (red spheres). The openings to the pores are blocked by $\alpha$-cyclodextrin $(\alpha-C D)$ based rotaxanes containing enzyme-cleavable stoppers. Cleavage of the stoppers by the appropriate enzyme releases the $\alpha$-CD macrocycles and allows the payload to diffuse from the pores.

containers (SCSNs). ${ }^{41}$ An SCSN consists of a [2]rotaxane tethered to the surface of a silica particle $(400 \mathrm{~nm}$ in diameter) containing hexagonally arranged $2 \mathrm{~nm}$ diameter pores. The [2]rotaxanes on the surface incorporate $\alpha$-cyclodextrin $(\alpha-C D)$ rings, each encircling an oligo(ethylene glycol) thread and fixed in place by a CuAAC reaction with a cleavable stopper. When intact, these rotaxanes efficiently trap guest molecules, such as the fluorescent probe rhodamine $B$, within the pores of the silica. However, hydrolysis of the adamantyl ester-containing stopper, catalyzed by porcine liver esterase, results in dethreading of the $\alpha-C D$ rings and diffusion of the guest molecules out of the pores, while noncleavable control stoppers or denatured samples of the enzyme showed no such evidence of guest molecule release. Because of the wide range of stoppering units that can be attached to the SCSN precursor, a multitude of snap-top systems with differentiated modes of activation can be prepared with relative ease. In addition to further developing the SCSN systems, we anticipate using the CUAAC reaction, carried out either in solution or through recently developed microcontact printing techniques, ${ }^{42}$ to incorporate new classes of electrochemically active mechanically interlocked compounds into molecular electronic devices.

\section{Conclusions}

As a result of investigating mild chemical transformations compatible with the tetracationic cyclophane $\mathrm{CBPQT}^{4+}$ that operate under either kinetic or thermodynamic control, we have developed template-directed synthetic protocols to provide well-defined donor-acceptor mechanically interlocked compounds with increased convergence and reaction efficiency. These complementary advances serve to make bistable [2]rotaxanes and bistable [2]catenanes of this type more compatible with a variety of new device architectures and aid and abet the efficient preparation of previously inaccessible compounds, including large well-defined mechanically interlocked compounds and polymers. These synthetic tools are indispensible as we seek to create complex systems with emergent properties ${ }^{43}$ and increasingly sophisticated functions, especially within device settings.

The collaboration was supported by the Semiconductor Research Corporation (SRC) and its Focus Centers on Functional Engineered Nanoarchitectonics (FENA) and Materials Structures and Devices (MSD), the Moletronics Program of the Defense Advanced Research Projects Agency (DARPA), and the Center for Nanoscale Innovation for Defense (CNID). J.M.S. gratefully acknowledges the National Science Foundation (NSF) for a Graduate Research Fellowship.

\section{BIOGRAPHICAL INFORMATION}

William R. Dichtel received his B.S. in chemistry in 2000 from MIT and his Ph.D. in 2005 from the University of California, Berkeley, under the supervision of Prof. Jean M. J. Fréchet. He was a research associate working jointly with Prof. J. Fraser Stoddart at the University of California, Los Angeles (UCLA), and Prof. James R. Heath at the California Institute of Technology from 2005 to 2008 and is currently an assistant professor in the Department of Chemistry and Chemical Biology at Cornell University.

Ognjen Š. Miljanić was born in Belgrade, Serbia, in 1978. He holds a Diploma degree from the University of Belgrade (2000) and a Ph. D. from the University of California, Berkeley (2005, with Professor K. Peter C. Vollhardt). After a postdoctoral stay at UCLA (2005-2008) with Professor J. Fraser Stoddart, Ognjen accepted a position as assistant professor at the University of Houston.

Wenyu Zhangwas born in Jiangsu, China, in 1983. He earned his B.S. in chemistry from Peking University, China, in 2005. He is currently a Ph.D. candidate in the Department of Chemistry and Biochemistry at UCLA under the direction of Professor Stoddart.

Jason Spruell earned his B.S. from the University of Alabama in 2005. Since then, he has pursued his Ph.D. in Chemistry with Pro- 
fessor Stoddart, beginning first of all at UCLA and now at Northwestern University.

Kaushik Patel received his B.S. in chemistry from Mercer University in 2002. He enrolled at UCLA in order to pursue graduate research with Professor Stoddart and moved to Northwestern University in late 2007.

Ivan Aprahamianreceived all his degrees (B.Sc., M.Sc., and Ph.D.) from the Hebrew University of Jerusalem, Israel. His Ph.D. research was conducted under the supervision of Profs. Mordecai Rabinovitz and Tuvia Sheradsky. He joined the Stoddart group (UCLA) as a postdoctoral researcher in 2005 and is presently an assistant professor at Dartmouth College.

James R. Heath is the Elizabeth W. Gilloon Professor and Professor of Chemistry at Caltech and Professor of Molecular and Medical Pharmacology at UCLA. He received a B.Sc. degree in 1984 (Baylor) and his Ph.D. in chemistry (Rice) in 1988. Heath was a Miller Fellow at the University of California, Berkeley, from 1988 to 1991 and a member of the Technical Staff at IBM Watson Laboratories from 1991 to 1994. In 1994, he joined the faculty at UCLA. He founded the California NanoSystems Institute (CNSI) in 2000 and served as its Director until he moved to Caltech in 2002.

Fraser Stoddartreceived his B.Sc. (1964) and Ph.D. (1966) degrees from Edinburgh University. In 1967, he went to Queen's University (Canada) as a National Research Council Postdoctoral Fellow and then, in 1970, to Sheffield University as an Imperial Chemical Industries (ICI) Research Fellow, before joining the academic staff there as a Lecturer in Chemistry. After spending a sabbatical (1978-1981) at the ICI Corporate Laboratory in Runcorn, he returned to a Readership at Sheffield in 1982. In 1990, he took up the Chair of Organic Chemistry at Birmingham University and was Head of the School of Chemistry there (1993-1997) before moving to UCLA as the Saul Winstein Professor of Chemistry in 1997. He was the Director of the CNSI from 2002 to 2007 and during that time held the Kavli Chair in NanoSystems Sciences. He joined the faculty at Northwestern University as a Board of Trustees Professor of Chemistry in January 2008.

\section{FOOTNOTES}

*To whom correspondence should be addressed. E-mail addresses: stoddart@ northwestern.edu; heath@caltech.edu.

\section{REFERENCES}

1 Green, J. E.; Choi, J. W.; Boukai, A.; Bunimovich, Y.; Johnston-Halperin, E.; Delonno, E.; Luo, Y.; Sheriff, B. A.; Xu, K.; Shin, Y. S.; Tseng, H.-R.; Stoddart, J. F.; Heath, J. R. A 160-Kilobit Molecular Electronic Memory Patterned at 10(11) Bits Per Square Centimetre. Nature 2007, 445, 414-417.

2 Ashton, P. R.; Goodnow, T. T.; Kaifer, A. E.; Reddington, M. V.; Slawin, A. M. Z.; Spencer, N.; Stoddart, J. F.; Vicent, C.; Williams, D. J. A [2]Catenane Made to Order. Angew. Chem., Int. Ed. Engl. 1989, 28, 1396-1399.

3 Choi, J. W.; Flood, A. H.; Steuerman, D. W.; Nygaard, S.; Braunschweig, A. B.; Moonen, N. N. P.; Laursen, B. W.; Luo, Y.; Delonno, E.; Peters, A. J.; Jeppesen, J. 0.; Xu, K.; Stoddart, J. F.; Heath, J. R. Ground-State Equilibrium Thermodynamics and Switching Kinetics of Bistable [2]Rotaxanes Switched in Solution, Polymer Gels, and Molecular Electronic Devices. Chem. Eur. J. 2006 12, 261-279.
4 Rostovtsev, V. V.; Green, L. G.; Fokin, V. V.; Sharpless, K. B. A Stepwise Huisgen Cycloaddition Process: Copper(I)-Catalyzed Regioselective "Ligation" of Azides and Terminal Alkynes. Angew. Chem., Int. Ed. 2002, 41, 2596-2599.

5 Tornoe, C. W.; Christensen, C.; Meldal, M. Peptidotriazoles on Solid Phase: $[1,2,3]$-Triazoles by Regiospecific Copper(I)-Catalyzed 1,3-Dipolar Cycloadditions of Terminal Alkynes to Azides. J. Org. Chem. 2002, 67, 30573064.

6 Dichtel, W. R.; Miljanić, O.Š.; Spruell, J. M.; Heath, J. R.; Stoddart, J. F. Efficient Templated Synthesis of Donor-Acceptor Rotaxanes Using Click Chemistry. J. Am. Chem. Soc. 2006, 128, 10388-10390.

7 Lutz, J. F. 1,3-Dipolar Cycloadditions of Azides and Alkynes: A Universal Ligation Tool in Polymer and Materials Science. Angew. Chem., Int. Ed. 2007, 46, 1018-1025.

8 Kolb, H. C.; Sharpless, K. B. The Growing Impact of Click Chemistry on Drug Discovery. Drug Discovery Today 2003, 8, 1128-1137.

9 Mobian, P.; Collin, J.-P.; Sauvage, J.-P. Efficient Synthesis of a Labile Copper(I)-Rotaxane Complex Using Click Chemistry. Tetrahedron Lett. 2006, 47, 4907-4909.

10 Aucagne, V.; Hanni, K. D.; Leigh, D. A.; Lusby, P. J.; Walker, D. B. Catalytic "Click" Rotaxanes: A Substoichiometric Metal-Template Pathway to Mechanically Interlocked Architectures. J. Am. Chem. Soc. 2006, 128, 21862187.

11 Miljanić, 0.S.; Dichtel, W. R.; Mortezaei, S.; Stoddart, J. F. Cyclobis(paraquat- $p$ phenylene)-Based [2]Catenanes Prepared by Kinetically Controlled Reactions Involving Alkynes. Org. Lett. 2006, 8, 4835-4838.

12 Miljanić, O. Š.; Dichtel, W. R.; Khan, S. I.; Mortezaei, S.; Heath, J. R.; Stoddart J. F. Structural and Co-Conformational Effects of Alkyne-Derived Subunits in Charged Donor-Acceptor 2 Catenanes. J. Am. Chem. Soc. 2007, 129, 82368246.

13 Siemsen, P.; Livingston, R. C.; Diederich, F. Acetylenic Coupling: A Powerful Tool in Molecular Construction. Angew. Chem., Int. Ed. 2000, 39, 2633-2657.

14 Rowan, S. J.; Cantrill, S. J.; Cousins, G. R. L.; Sanders, J. K. M.; Stoddart, J. F. Dynamic Covalent Chemistry. Angew. Chem., Int. Ed. 2002, 41, 898-952.

15 Corbett, P. T.; Leclaire, J.; Vial, L.; West, K. R.; Wietor, J. L.; Sanders, J. K. M.; Otto, S. Dynamic Combinatorial Chemistry. Chem. Rev. 2006, 106, 3652-3711.

16 Miljanić, 0. Š.; Stoddart, J. F. Dynamic Donor-Acceptor [2]Catenanes. Proc. Natl. Acad. Sci. U.S.A. 2007, 104, 12966-12970.

17 D'Acerno, C.; Doddi, G.; Ercolani, G.; Mencarelli, P. Template Effects and Kinetic Selection in the Self-Assembly of Crown Ether Cyclobis(Paraquat-PPhenylene) 2 Catenanes - Effect of the 1,4-Dioxybenzene and 1,5Dioxynaphthalene Units. Chem. Eur. J. 2000, 6, 3540-3546.

18 Asakawa, M.; Ashton, P. R.; Balzani, V.; Credi, A.; Hamers, C.; Mattersteig, G.; Montalti, M.; Shipway, A. N.; Spencer, N.; Stoddart, J. F.; Tolley, M. S.; Venturi, M.; White, A. J. P.; Williams, D. J. A Chemically and Electrochemically Switchable [2]Catenane Incorporating a Tetrathiafulvalene Unit. Angew. Chem., Int. Ed. 1998, 37, 333-337.

19 Asakawa, M.; Ashton, P. R.; Menzer, S.; Raymo, F. M.; Stoddart, J. F.; White, A. J. P.; Williams, D. J. Cyclobis(Paraquat-4,4'-Biphenylene) - an Organic Molecular Square. Chem. Eur. J. 1996, 2, 877-893.

20 Patel, K.; Miljanić,0. Š.; Stoddart, J. F. Iodide-Catalysed Self-Assembly of DonorAcceptor [3]Catenanes. Chem. Commun. 2008, 1853-1855.

21 Liu, Y.; Flood, A. H.; Bonvallett, P. A.; Vignon, S. A.; Northrop, B. H.; Tseng, H.-R.; Jeppesen, J. O.; Huang, T. J.; Brough, B.; Baller, M.; Magonov, S.; Solares, S. D.; Goddard, W. A.; Ho, C. M.; Stoddart, J. F. Linear Artificial Molecular Muscles. J. Am. Chem. Soc. 2005, 127, 9745-9759.

22 Aucagne, V.; Leigh, D. A. Chemoselective Formation of Successive Triazole Linkages in One Pot: "Click-Click" Chemistry. Org. Lett. 2006, 8, 4505-4507.

23 Orsini, A.; Viterisi, A.; Bodlenner, A.; Weibel, J. M.; Pale, P. A Chemoselective Deprotection of Trimethylsilyl Acetylenes Catalyzed by Silver Salts. Tetrahedron Lett. 2005, 46, 2259-2262.

24 Spruell, J. M.; Dichtel, W. R.; Heath, J. R.; Stoddart, J. F. A One-Pot Synthesis of Constitutionally Unsymmetrical Rotaxanes Using Sequential $\mathrm{Cu}(\mathrm{I})$-Catalyzed Azide-Alkyne Cycloadditions. Chem. Eur. J. 2008, 14, 1468-1477.

25 Braunschweig, A. B.; Dichtel, W. R.; Miljanić, O. Š.; Olson, M. A.; Spruell, J. M.; Khan, S. I.; Heath, J. R.; Stoddart, J. F. Modular Synthesis and Dynamics of a Variety of Donor-Acceptor Interlocked Compounds Prepared by Click Chemistry. Chem. Asian J. 2007, 2, 634-647.

26 Kang, S. S.; Vignon, S. A.; Tseng, H.-R.; Stoddart, J. F. Molecular Shuttles Based on Tetrathiafulvalene Units and 1,5-Dioxynaphthalene Ring Systems. Chem. Eur. J. 2004, 10, 2555-2564.

27 Aprahamian, I.; Dichtel, W. R.; Ikeda, T.; Heath, J. R.; Stoddart, J. F. A Clicked Bistable [2]Rotaxane. Org. Lett. 2007, 9, 1287-1290. 
28 Steuerman, D. W.; Tseng, H.-R.; Peters, A. J.; Flood, A. H.; Jeppesen, J. 0.; Nielsen, K. A.; Stoddart, J. F.; Heath, J. R. Molecular-Mechanical Switch-Based Solid-State Electrochromic Devices. Angew. Chem., Int. Ed. 2004, 43, 6486-6491.

29 Tseng, H.-R.; Wu, D. M.; Fang, N. X. L.; Zhang, X.; Stoddart, J. F. The Metastability of an Electrochemically Controlled Nanoscale Machine on Gold Surfaces. ChemPhysChem 2004, 5, 111-116.

30 Aprahamian, I.; Yasuda, T.; Ikeda, T.; Saha, S.; Dichtel, W. R.; Isoda, K.; Kato, T.; Stoddart, J. F. A Liquid-Crystalline Bistable [2]Rotaxane. Angew. Chem., Int. Ed. 2007, 46, 4675-4679.

31 Eelkema, R.; Pollard, M. M.; Vicario, J.; Katsonis, N.; Ramon, B. S.; Bastiaansen, C. W. M.; Broer, D. J.; Feringa, B. L. Nanomotor Rotates Microscale Objects. Nature 2006, 440, 163-163.

32 Zhang, W.; Dichtel, W. R.; Steig, A. Z.; Benítez, D.; Gimzewski, J. K.; Heath, J. R.; Stoddart, J. F. Folding in a Donor-Acceptor Polyrotaxane Using Secondary Noncovalent Interactions. Proc. Natl. Acad. Sci. U.S.A. 2008, 105, 6514-6519.

33 Wenz, G.; Han, B. H.; Müller, A. Cyclodextrin Rotaxanes and Polyrotaxanes. Chem. Rev. 2006, 106, 782-817.

34 Whang, D.; Jeon, Y. M.; Heo, J.; Kim, K. Self-Assembly of a Polyrotaxane Containing a Cyclic "Bead" in Every Structural Unit in the Solid State: Cucurbituril Molecules Threaded on a One-Dimensional Coordination Polymer. J. Am. Chem. Soc. 1996 $118,11333-11334$

35 Lokey, R. S.; Iverson, B. L. Synthetic Molecules That Fold into a Pleated Secondary Structure in Solution. Nature 1995, 375, 303-305.
36 Appella, D. H.; Christianson, L. A.; Klein, D. A.; Powell, D. R.; Huang, X. L.; Barchi, J. J.; Gellman, S. H. Residue-Based Control of Helix Shape in BetaPeptide Oligomers. Nature 1997, 387, 381-384.

37 Hill, D. J.; Moore, J. S. Helicogenicity of Solvents in the Conformational Equilibrium of Oligo( $m$-phenylene ethynylene)s: Implications for Foldamer Research. Proc. Natl. Acad. Sci. U.S.A. 2002, 99, 5053-5057.

38 Lummerstorfer, T.; Hoffmann, H. Click Chemistry on Surfaces: 1,3-Dipolar Cycloaddition Reactions of Azide-Terminated Monolayers on Silica. J. Phys. Chem. B 2004, 108, 3963-3966.

39 Collman, J. P.; Devaraj, N. K.; Chidsey, C. E. D. "Clicking" Functionality onto Electrode Surfaces. Langmuir 2004, 20, 1051-1053.

40 Rohde, R. D.; Agnew, H. D.; Yeo, W. S.; Bailey, R. C.; Heath, J. R. A NonOxidative Approach toward Chemically and Electrochemically Functionalizing Si(111). J. Am. Chem. Soc. 2006, 128, 9518-9525.

41 Patel, K.; Angelos, S.; Dichtel, W. R.; Coskun, A.; Yang, Y.-W.; Zink, J. I.; Stoddart, J. F. Enzyme-Responsive Snap-Top Covered Silica Nanocontainers. J. Am. Chem. Soc. 2008, 130, 2382-2383.

42 Rozkiewicz, D. I.; Janczewski, D.; Verboom, W.; Ravoo, B. J.; Reinhoudt, D. N. "Click" Chemistry by Microcontact Printing. Angew. Chem., Int. Ed. 2006, 45, 5292-5296.

43 Aprahamian, I.; Olsen, J.-C.; Trabolsi, A.; Stoddart, J. F. Tetrathiafulvalene Radical Cation Dimerization in a Bistable Tripodal [4]Rotaxane. Chem. Eur. J. 2008, 14, 3889-3895. 